\title{
Wilson fermions at fine lattice spacings: scale setting, pion form factors and $(g-2)_{\mu}$
}

\section{Bastian B. Brandt, Stefano Capitani, Dalibor Djukanovic, Georg von Hippel, Benjamin Jäger, Bastian Knippschild \\ Institut für Kernphysik, University of Mainz, Becher Weg 45, 55099 Mainz, Germany}

Michele Della Morte, Hartmut Wittig*

Institut für Kernphysik and Helmholtz. Institute Mainz, University of Mainz, Becher Weg 45, 55099 Mainz, Germany

E-mail: wittig@kph.uni-mainz.de

\section{Andreas Jüttner}

Physics Department, TH Unit, 1211 Geneva 23, Switzerland

We present an update on our on-going project to compute hadronic observables for $N_{\mathrm{f}}=2$ flavours of $\mathrm{O}(a)$ improved Wilson fermions at small lattice spacings. The procedure to determine the lattice scale via the mass of the Omega baryon is described. Furthermore we present preliminary results for the pion form factor computed using twisted boundary conditions, and report on the implementation of a novel approach to determine the contribution of the hadronic vacuum polarisation to the anomalous magnetic moment of the muon.

The XXVIII International Symposium on Lattice Field Theory, Lattice2010

June 14-19, 2010

Villasimius, Italy

\footnotetext{
* Speaker.
} 


\section{Introduction}

Studies of structural properties of mesons and baryons, encoded in their electromagnetic form factors, are the subject of many recent lattice calculations. However, systematic uncertainties arising from a variety of sources appear to be much larger than for simpler quantities such as hadron masses and decay constants. As part of the CLS project ${ }^{1}$ a set of ensembles is being generated, which will allow for a thorough investigation of systematic effects, such as lattice artefacts, finitevolume effects, and extrapolations in the light quark masses. The CLS project is based on proven and conceptually simple technology: simulations are performed using non-perturbatively $\mathrm{O}(a)$ improved Wilson quarks and the Wilson plaquette action. In order to preserve the local structure of the lattice action, the link variables remain unsmeared. We employ the deflation-accelerated DD-HMC algorithm [1] for $N_{\mathrm{f}}=2$ flavours of light quarks.

A potentially severe problem for any lattice simulation near the continuum limit is the observed sharp rise in the autocorrelation time of the topological charge [2]. This important issue has been addressed in several papers [3,4] and contributions to this conference [5]. In spite of the progress made in identifying the causes of this phenomenon, a rigorous and effective solution is still elusive. Therefore we have restricted the calculation of observables to a range of bare couplings for which the problem of very long autocorrelation times in the topological charge is not observed. A compilation of these ensembles is shown in Table 1. At our smallest value of the lattice spacing, $a \approx 0.05 \mathrm{fm}$ (i.e. at $\beta=5.5$ ), where the problem is expected to be most severe, we have checked explicitly that the topological charge fluctuates around zero at a sizeable rate and produces a reasonably symmetric distribution [6]. We can therefore take confidence that the composition of our ensembles is not strongly biased and that our statistical errors are reliable.

In this note we report on preliminary results for the pion electromagnetic form factor. ${ }^{2}$ Another issue which has received a lot of attention recently, is the hadronic vacuum polarisation contribution to the muon's anomalous magnetic moment, $a_{\mu}^{\text {had }}$. Lattice calculations of this quantity involve the determination of the vacuum polarisation amplitude, which, like hadronic form factors, depends on

\begin{tabular}{ccccccc}
\hline \hline$\beta$ & $a[\mathrm{fm}]$ & lattice & $L[\mathrm{fm}]$ & $\#$ masses & $m_{\pi} L$ & Labels \\
\hline 5.20 & 0.08 & $64 \times 32^{3}$ & 2.6 & 4 masses & $4.8-9.0$ & A1-A4 \\
\hline 5.30 & 0.07 & $48 \times 24^{3}$ & 1.7 & 3 masses & $4.6-7.9$ & D1-D3 \\
5.30 & 0.07 & $64 \times 32^{3}$ & 2.2 & 3 masses & $4.7-7.9$ & E3-E5 \\
5.30 & 0.07 & $96 \times 48^{3}$ & 3.4 & 2 masses & $5.0,4.2$ & F6, F7 \\
\hline 5.50 & 0.05 & $96 \times 48^{3}$ & 2.5 & 3 masses & $5.3-7.7$ & N3- N5 \\
5.50 & 0.05 & $128 \times 64^{3}$ & 3.4 & 1 mass & 4.7 & O6 \\
\hline \hline
\end{tabular}

Table 1: Simulation parameters and approximate values for the lattice scale and pion masses for those ensembles which show an acceptable tunnelling rate of the topological charge. The preliminary results presented in theses proceedings are based on the ensembles labelled "N", "E" and "F6".

\footnotetext{
${ }^{1}$ https://twiki.cern.ch/twiki/bin/view/CLS/WebHome

${ }^{2}$ Form factor calculations for baryons are presented in another contribution to this conference [7].
} 

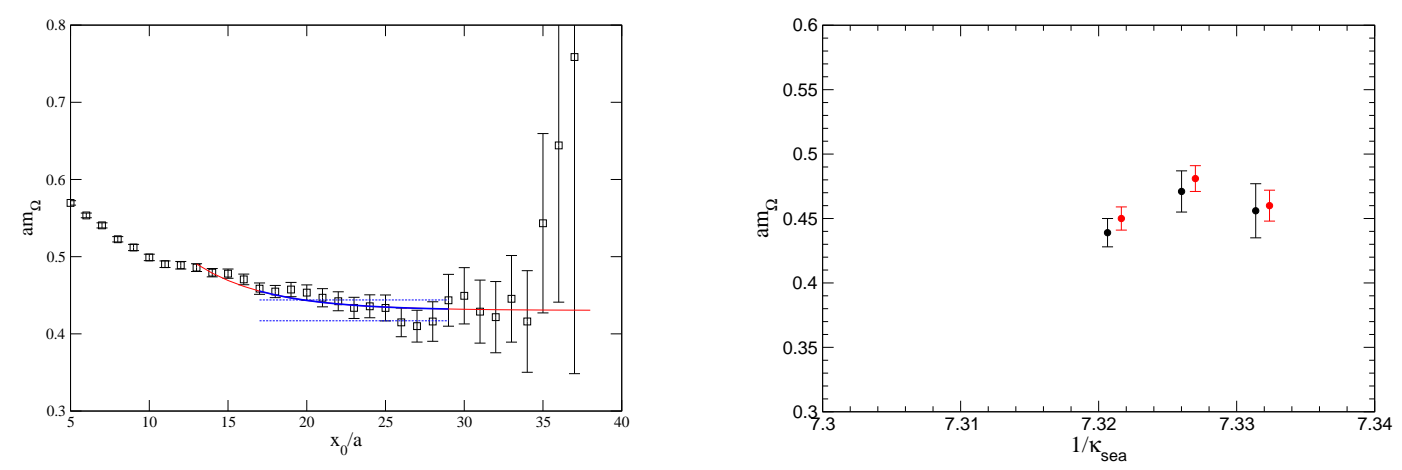

Figure 1: Left: the effective mass in the $\Omega$-channel for the ensemble N5 $\left(m_{\pi} \approx 410 \mathrm{MeV}\right)$; Right: Sea quark mass dependence of $m_{\Omega}$ for datasets N3 - N5. Red and black symbols denote the results from correlated and uncorrelated fits, respectively.

a momentum variable. Therefore, we discuss both the calculation of mesonic form factors and the determination of $a_{\mu}^{\text {had }}$ in these proceedings.

\section{Setting the scale}

Studying the scaling properties of observables and expressing dimensionful quantities in physical units requires the computation of a reference quantity which sets the scale. The mass of the $\Omega$ baryon has emerged as a good candidate, since the $\Omega$ is stable in QCD, and its valence sector consists entirely of strange quarks. Moreover, ChPT studies [8] suggest a simple functional form for chiral extrapolations in the sea quark mass.

In order to determine the masses of pseudoscalar and vector mesons, as well as octet and decuplet baryons, we used Jacobi-smeared sources [9], supplemented by HYP-smeared [10] link variables. In the pseudoscalar channel long and stable plateaus were observed, which allowed for a reliable determination of the mass via single-cosh fits to the correlation functions. In the vector channel and for baryons, the unambiguous identification of the ground state turned out to be more difficult. We therefore employed the procedure of [11], which is based on an ansatz that includes contributions from the first excited state. In Fig. 1 we show a typical effective mass plot in the $\Omega$-channel together with the fit result.

In order to determine $m_{\Omega}$ an interpolation in the valence quark mass must be performed. This requires knowledge of the hopping parameter, $\kappa_{s}$, which corresponds to the bare strange quark mass. We have determined $\kappa_{s}$ using the procedure described in $[6,12]$ : Denoting the generic nondegenerate pseudoscalar and vector mesons by $m_{K}$ and $m_{K}^{*}$, respectively, we interpolate the mass ratio $\left(m_{K} / m_{K}^{*}\right)^{2}$ as a function of $\left(a m_{K}\right)^{2}$ to the experimental value $m_{K} / m_{K}^{*}=0.554$. This yields the value of $\kappa_{s}$ at each value of the sea quark mass. In a second step one interpolates $a m_{\Omega}$ in the valence quark mass to the value of $\kappa_{s}$. The resulting estimates of $a m_{\Omega}$ as a function of $1 / \kappa_{\text {sea }}$ are shown for $\beta=5.5$ in the right panel of Fig. 1. A mild dependence on the sea quark mass is observed. At this stage we have not yet attempted a chiral extrapolation in the sea quark mass to the physical value of $m_{\pi}$. In order to set the scale at $\beta=5.5$ we therefore take the result for $a m_{\Omega}$ at the smallest sea quark, which yields $a_{\Omega}=0.053(1) \mathrm{fm}$. 
Our production runs for baryonic two-point functions on the other ensembles are not yet completed. In order to compare form factors computed at different lattice spacings consistently, we employ a simple scaling relation, i.e.

$$
\left.a_{\Omega}\right|_{\beta=5.3}=\left.\left(\frac{\left.a_{\mathrm{ref}}\right|_{\beta=5.3}}{\left.a_{\mathrm{ref}}\right|_{\beta=5.5}}\right) a_{\Omega}\right|_{\beta=5.5} .
$$

Here the scale $a_{\text {ref }}$ is defined via the kaon mass $a m_{K}$, evaluated at the (unphysical) reference point where $m_{\pi} / m_{K}=0.85$ [12], which can be reached without performing a chiral extrapolation. At $\beta=5.3$ this yields $a_{\text {ref }}=0.0784(10) \mathrm{fm}$ [12], while at $\beta=5.5$ one obtains $a_{\text {ref }}=0.0603(15) \mathrm{fm} \mathrm{[6]}$. Our result for $a_{\Omega}$ at $\beta=5.5$ and the scaling relation eq. (2.1) imply

$$
\left.a_{\Omega}\right|_{\beta=5.5}=0.053(1) \mathrm{fm},\left.\quad a_{\Omega}\right|_{\beta=5.3}=0.069(2) \mathrm{fm} .
$$

At $\beta=5.5$ the value of $a_{\Omega}$ is smaller by more than $10 \%$ compared to $a_{\text {ref }}$, which we used previously to convert our pion masses on the "N"-lattices into physical units (see Table 2 of ref. [6]). Using the value in eq. (2.2) the pion masses change to $m_{\pi}=600,510$ and $410 \mathrm{MeV}$ on N3, N4 and N5, respectively.

\section{The pion electromagnetic form factor}

The electromagnetic form factor, defined by

$$
\left\langle\pi^{+}\left(\vec{p}_{f}\right)\left|\frac{2}{3} \bar{u} \gamma_{\mu} u-\frac{1}{3} \bar{d} \gamma_{\mu} d\right| \pi^{+}\left(\vec{p}_{i}\right)\right\rangle=\left(p_{f}+p_{i}\right)_{\mu} f_{\pi}\left(q^{2}\right),
$$

where $q=p_{f}-p_{i}$ is the momentum transfer, encodes the distribution of electric charge inside the pion. Of particular interest is the charge radius, $\left\langle r_{\pi}^{2}\right\rangle$, which is derived from the pion form factor at vanishing momentum transfer, i.e.

$$
f_{\pi}\left(q^{2}\right)=1-\frac{1}{6}\left\langle r_{\pi}^{2}\right\rangle q^{2}+\mathrm{O}\left(q^{4}\right) \quad \Rightarrow \quad\left\langle r_{\pi}^{2}\right\rangle=\left.6 \frac{\mathrm{d} f_{\pi}\left(q^{2}\right)}{\mathrm{d} q^{2}}\right|_{q^{2}=0} .
$$

Lattice calculations of mesonic matrix elements are technically simpler than the corresponding quantities for the nucleon. Furthermore, the pion electromagnetic form factor receives no contributions from quark-disconnected diagrams. This opens the possibility to perform a precision test of lattice QCD, by comparing lattice estimates for $\left\langle r_{\pi}^{2}\right\rangle$ to the experimentally determined value. However, owing to the finite spatial volume the accessible range of momentum transfers $Q^{2}=-q^{2}$ is severely constrained, which presents a major obstacle for precise lattice determinations of $\left\langle r_{\pi}^{2}\right\rangle$. We have therefore employed flavour-twisted boundary conditions [14] in the valence sector, such that the momentum transfer satisfies [15]

$$
-Q^{2} \equiv q^{2}=\left(p_{f}-p_{i}\right)^{2}=\left(E_{\pi}\left(\vec{p}_{f}\right)-E_{\pi}\left(\vec{p}_{i}\right)\right)^{2}-\left[\left(\vec{p}_{f}+\frac{\vec{\theta}_{f}}{L}\right)-\left(\vec{p}_{i}+\frac{\vec{\theta}_{i}}{L}\right)\right]^{2} .
$$

We tuned the twist angles $\vec{\theta}_{i}, \vec{\theta}_{f}$ so as to achieve a particularly fine momentum resolution near $Q^{2}=$ 0 . In order to improve the statistical signal we used stochastic $Z_{2} \times Z_{2}$ sources in the calculation 

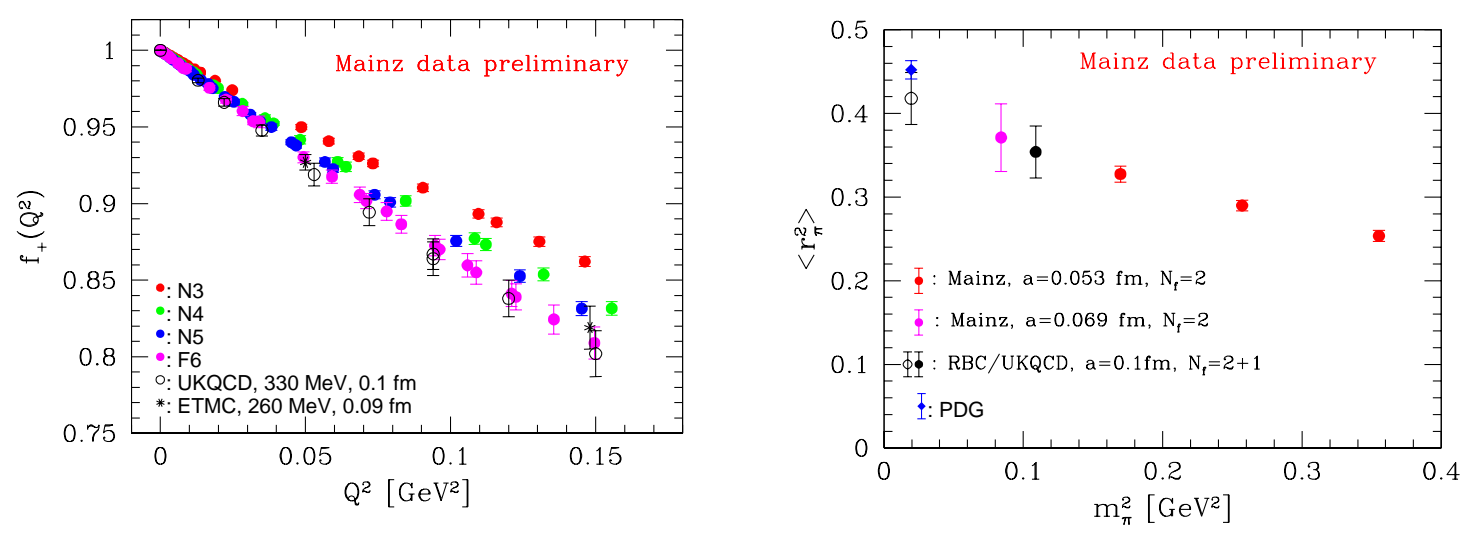

Figure 2: Left: pion form factor computed for a range of pion masses compared to the results of [16,17]; Right: the charge radius as a function of the pion mass, compared with the results of the $2+1$-flavour simulation from [16].

of quark propagators. As in ref. [16] the pion form factor was extracted from a suitable ratio of correlators in which the renormalisation factor of the local vector current, $Z_{\mathrm{V}}$, drops out.

In Fig. 2 we show our results for the pion electromagnetic form factor as a function of the squared Euclidean momentum transfer for the data sets N3, N4, N5 and F6. The corresponding pion masses in $\mathrm{MeV}$ are 600, 510, 410 and 290, respectively. Twisted boundary conditions were also employed in refs. [16,17], which are shown together with our data. The much larger number of data points near $Q^{2}=0$ allows us to perform a very accurate and largely model-independent determination of the pion charge radius, by computing the linear slope of $f_{\pi}\left(Q^{2}\right)$ in the vicinity of $Q^{2}=0$. In this way our estimate of the pion charge radius does not rely on a particular ansatz, such as vector dominance. As can be seen from the right panel in Fig. 2 we obtain statistically very precise results for $\left\langle r_{\pi}^{2}\right\rangle$, in particular on the ensembles at $\beta=5.5$. Statistics at our lightest quark mass $\left(\beta=5.3\right.$ ) will be increased. Our preliminary results for $\left\langle r_{\pi}^{2}\right\rangle$ are consistent with the determination of ref. [16], which uses $N_{\mathrm{f}}=2+1$ flavours of domain wall quarks, and the trend in our lattice data compares favourably with the experimentally determined value.

\section{Hadronic vacuum polarisation contribution to $(g-2) \mu$}

The anomalous magnetic moment of the muon, $a_{\mu} \equiv \frac{1}{2}(g-2)_{\mu}$, is among the most precisely measured quantities. Assuming the validity of the Standard Model, the experimental value for $a_{\mu}$ differs from the theoretical prediction at the level of 3.2 standard deviations [18]. An important ingredient is the leading hadronic contribution to vacuum polarisation, $a_{\mu}^{\text {had }}$. This quantity is normally determined via a semi-phenomenological approach based on the evaluation of a dispersion integral containing experimentally measured hadronic cross sections. Given the importance of $a_{\mu}$ for new physics searches, a first-principles determination of $a_{\mu}^{\text {had }}$ is highly desirable. Following refs. $[19,20], a_{\mu}^{\text {had }}$ can be computed on the lattice via the convolution integral

$$
a_{\mu}^{\text {had }}=4 \pi^{2}\left(\frac{\alpha}{\pi}\right)^{2} \int_{0}^{\infty} \mathrm{d} Q^{2} f\left(Q^{2}\right)\left\{\Pi\left(Q^{2}\right)-\Pi(0)\right\},
$$



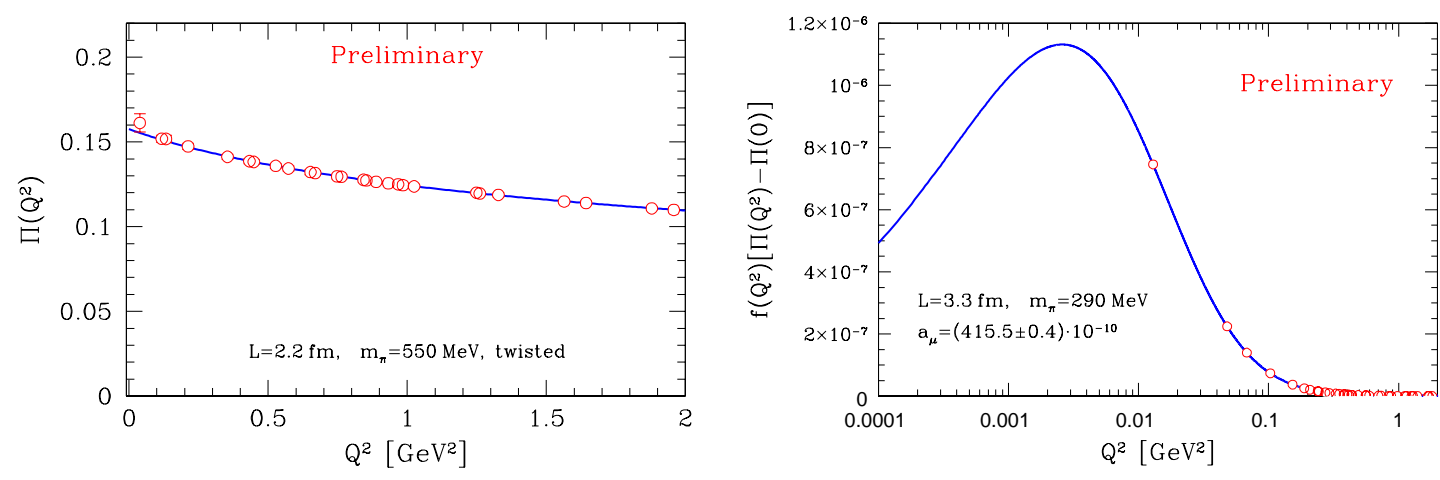

Figure 3: Left: the vacuum polarisation amplitude computed at $\beta=5.3, L \simeq 2.2 \mathrm{fm}, m_{\pi} \approx 550 \mathrm{MeV}$, using twisted boundary conditions. The solid curve is a Padé fit to the data; Right: the integrand of the convolution integral on the "F6" lattice. The area underneath the solid curve yields the value of $a_{\mu}^{\text {had }}$.

where $\Pi\left(Q^{2}\right)$ is related to the vacuum polarisation tensor $\Pi_{\mu v}$, i.e.

$$
\Pi_{\mu v}(Q)=\int \mathrm{d}^{4} x \mathrm{e}^{i q \cdot(x-y)}\left\langle J_{\mu}(x) J_{v}(y)\right\rangle \equiv\left(q_{\mu} q_{v}-g_{\mu v} q^{2}\right) \Pi\left(q^{2}\right) .
$$

Here $J_{\mu}$ denotes the flavour-singlet vector current. A well-known problem is that the convolution function $f\left(Q^{2}\right)$ is strongly peaked for momenta near $m_{\mu}$, which is an order of magnitude smaller than what can conventionally be realised on current lattice sizes. Twisted boundary conditions cannot be used in a straightforward manner, since the current-current correlator contains quarkdisconnected diagrams, for which the effect of the twist cancels.

To tackle this problem we have implemented the strategy outlined in [21]: The key observation is that the continuum limit exists for the individual quark-disconnected and connected contributions to П. Moreover, the connected part can be re-interpreted in terms of flavour-non-singlet correlators, which are easily computed using twisted boundary conditions in the standard fashion. ChPT at NLO also predicts that the contribution from disconnected diagrams is suppressed by a factor $-1 / 10$ relative to the connected ones [21].

Preliminary results for the vacuum polarisation amplitude extracted from the connected correlator in the two-flavour case are shown in Fig. 3. Twisted boundary conditions produce a much larger density of $Q^{2}$ values, which greatly stabilises the extrapolation to $Q^{2}=0$ and the extraction of $\Pi(0)$, which enters eq. (4.1). Furthermore, owing to the larger reach in $Q^{2}$ the integrand of the convolution integral is much more tightly constrained (c.f. Fig. 3, right panel). Our lattice estimates for $a_{\mu}^{\text {had }}$ computed for $N_{\mathrm{f}}=2$ and also in the theory containing a quenched strange quark will appear elsewhere [22]. Eventually we will also include quark-disconnected diagrams, computed for the usual Fourier-momenta only, in order to verify that their contribution is indeed suppressed.

\section{Summary and outlook}

The ensembles generated as part of the CLS project allow for a comprehensive investigation of systematic effects for a variety of hadronic observables. Our fine lattice resolution will enable reliable continuum extrapolations and will prove useful to explore hadronic form factors at large momentum transfers. Twisted boundary conditions are indispensable for an accurate determination 
of the pion form factor. Their use can also be extended to lattice determinations of $a_{\mu}^{\text {had }}$, for which a straightforward application of this technique is not obvious.

Acknowledgments: We thank our colleagues within the CLS project for sharing gauge ensembles. Calculations of correlation functions were performed on the dedicated QCD platform "Wilson" at the Institute for Nuclear Physics, University of Mainz. This work is supported by DFG (SFB443), GSI, and the Research Center EMG funded by Forschungsinitiative Rheinland-Pfalz.

\section{References}

[1] M. Lüscher, Comput. Phys. Commun. 165 (2005) 199, hep-lat/0409106; JHEP 12 (2007) 011, arXiv:0710.5417.

[2] L. Del Debbio, H. Panagopoulos and E. Vicari, JHEP 08 (2002) 044, hep-th/0204125; D.J. Antonio et al., Phys. Rev. D77 (2008) 014509, arXiv:0705.2340; S. Schaefer, R. Sommer and F. Virotta, PoS LAT2009 032, arXiv:0910.1465. A. Bazavov et al., Phys. Rev. D81 (2010) 114501, arXiv:1003.5695.

[3] M. Lüscher, Commun. Math. Phys. 293 (2010) 899, arXiv:0907.5491; JHEP 08 (2010) 071, arXiv: 1006.4518.

[4] S. Schaefer, R. Sommer and F. Virotta, Critical slowing down and error analysis in lattice QCD simulations, arXiv:1009.5228.

[5] M. Lüscher, Topology, the Wilson flow and the HMC algorithm, arXiv:1009.5877.

[6] S. Capitani et al., PoS LAT2009 095, arXiv:0910.5578.

[7] B. Knippschild, these proceedings.

[8] B.C. Tiburzi and A. Walker-Loud, Nucl. Phys. A764 (2006) 274, hep-lat/0501018.

[9] UKQCD Collaboration (C.R. Allton et al.) Phys. Rev. D47 (1993) 5128, hep-lat/9303009.

[10] A. Hasenfratz and F. Knechtli, Phys. Rev. D64 (2001) 034504, hep-lat/0103029.

[11] L. Del Debbio et al., JHEP 02 (2007) 082, hep-lat/0701009.

[12] L. Del Debbio et al., JHEP 02 (2007) 056, hep-lat/0610059.

[13] R. Sommer, Nucl. Phys. B411 (1994) 839, hep-lat/9310022.

[14] P.F. Bedaque, Phys. Lett. B593 (2004) 82, nucl-th/0402051; G.M. de Divitiis, R. Petronzio and N. Tantalo, Phys. Lett. B595 (2004) 408, hep-lat/0405002; C.T. Sachrajda and G. Villadoro, Phys. Lett. B609 (2005) 73, hep-lat/0411033; J.M. Flynn, A. Jüttner and C.T. Sachrajda, Phys. Lett. B632 (2006) 313, hep-lat/0506016.

[15] P.A. Boyle et al., JHEP 05 (2007) 016, hep-lat/0703005.

[16] P.A. Boyle et al., JHEP 07 (2008) 112, arXiv:0804.3971.

[17] R. Frezzotti, V. Lubicz and S. Simula, Phys. Rev. D79 (2009) 074506, arXiv:0812.4042.

[18] F. Jegerlehner and A. Nyffeler, Phys. Rept. 477 (2009) 1, arXiv:0902.3360.

[19] E. de Rafael, Phys. Lett. B322 (1994) 239, hep-ph/9311316.

[20] T. Blum, Phys. Rev. Lett. 91 (2003) 052001, hep-lat/0212018.

[21] A. Jüttner and M. Della Morte, PoS LAT2009 143, arXiv:0910.3755; Quark disconnected diagrams in chiral perturbation theory, arXiv:1009.3783.

[22] B. Jäger, A. Jüttner, M. Della Morte and H. Wittig, in preparation. 